\title{
Drosophila Temperature Preference Rhythms: An Innovative Model to Understand Body Temperature Rhythms
}

\author{
Tadahiro Goda ${ }^{1, *}$ and Fumika N. Hamada $1,2,3, *$ \\ 1 Visual Systems Group, Abrahamson Pediatric Eye Institute, Division of Pediatric Ophthalmology, \\ Cincinnati Children's Hospital Medical Center, 3333 Burnet Avenue, Cincinnati, OH 45229, USA \\ 2 Division of Developmental Biology, Cincinnati Children's Hospital Medical Center, Cincinnati, \\ OH 45229, USA \\ 3 Department of Ophthalmology, College of Medicine, University of Cincinnati, Cincinnati, OH 45229, USA \\ * Correspondence: Tadahiro.goda@cchmc.org (T.G.); Fumika.hamada@cchmc.org (F.N.H.); \\ Tel.: (513) 803-1662 (F.N.H.); Fax: (513) 803-0740 (F.N.H.)
}

Received: 1 March 2019; Accepted: 15 April 2019; Published: 23 April 2019

\begin{abstract}
Human body temperature increases during wakefulness and decreases during sleep. The body temperature rhythm (BTR) is a robust output of the circadian clock and is fundamental for maintaining homeostasis, such as generating metabolic energy and sleep, as well as entraining peripheral clocks in mammals. However, the mechanisms that regulate BTR are largely unknown. Drosophila are ectotherms, and their body temperatures are close to ambient temperature; therefore, flies select a preferred environmental temperature to set their body temperature. We identified a novel circadian output, the temperature preference rhythm (TPR), in which the preferred temperature in flies increases during the day and decreases at night. TPR, thereby, produces a daily BTR. We found that fly TPR shares many features with mammalian BTR. We demonstrated that diuretic hormone 31 receptor (DH31R) mediates Drosophila TPR and that the closest mouse homolog of DH31R, calcitonin receptor (Calcr), is essential for mice BTR. Importantly, both TPR and BTR are regulated in a distinct manner from locomotor activity rhythms, and neither DH31R nor Calcr regulates locomotor activity rhythms. Our findings suggest that DH31R/Calcr is an ancient and specific mediator of BTR. Thus, understanding fly TPR will provide fundamental insights into the molecular and neural mechanisms that control BTR in mammals.
\end{abstract}

Keywords: body temperature rhythms; Drosophila; DH31R; DH31; PDF; PDFR; circadian rhythms; locomotor activity rhythms; Calcr; Calcitonin receptor

\section{Introduction}

Most organisms exhibit approximately 24 -h cycles of physiological and behavioral activity $[1,2]$. In humans, body temperature fluctuates by approximately $1{ }^{\circ} \mathrm{C}$ over the course of a day, increasing gradually during wakefulness and decreasing during sleep (Figure 1A) [3]. The daily fluctuation of body temperature (body temperature rhythm: BTR) is a robust output of the circadian clock [4-6]. BTRs are associated with other physiological functions, such as metabolism and sleep [7-12], and play an important role in regulating the entrainment of the peripheral clocks located in the lungs and liver $[11,13]$. However, the molecular and neural mechanisms by which the circadian clock regulates BTRs remain largely unclear. The fruit fly Drosophila melanogaster is a versatile model organism with powerful and sophisticated genetic toolkits and has been used to clarify complex biological systems, including circadian rhythms and sleep. In 2017, the Nobel Prize in Physiology or Medicine was given to Drs. Jeffrey C. Hall, Michael Rosbash, and Michael W. Young for their discoveries of mechanisms 
regulating circadian rhythms using Drosophila. We first asked whether Drosophila can be used as a model organism for BTRs. We found that flies exhibit robust temperature preference behavior and that their preferred temperature fluctuates over a 24-h period. Because flies are small ectotherms and their body temperatures are very close to the ambient temperatures in the environments they choose, this fluctuation in preferred temperature can give rise to a BTR in flies $[7,14-18]$. The evidences in humans and rats suggest that BTRs are controlled separately from locomotor activity rhythms [7,19-22]. We found in Drosophila that BTR is controlled separately from the locomotor activity rhythms as well [18]. Furthermore, we recently showed that a G-protein-coupled receptor (GPCR), the calcitonin receptor, modulates BTRs but not locomotor activity rhythms in both mammals and flies [23]. Thus, our data suggest that there are evolutionarily conserved mechanisms that contribute to the circadian regulation of body temperature in mammals and flies. Here, we describe our current knowledge of the regulatory mechanisms underlying BTRs in Drosophila and mice and discuss how Drosophila can be a model organism for BTRs.

(A)

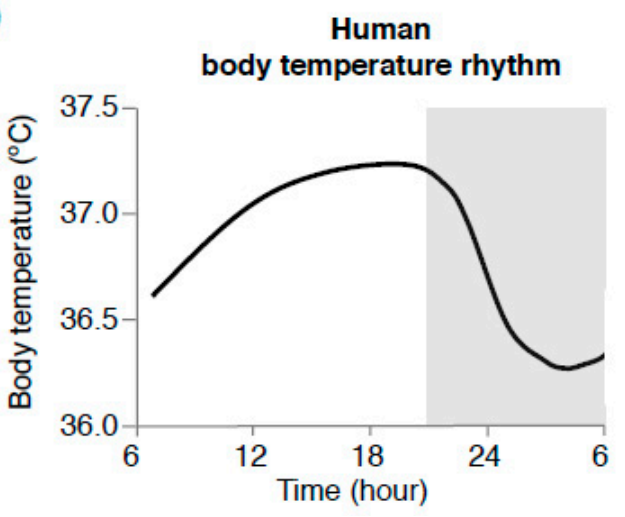

(B)

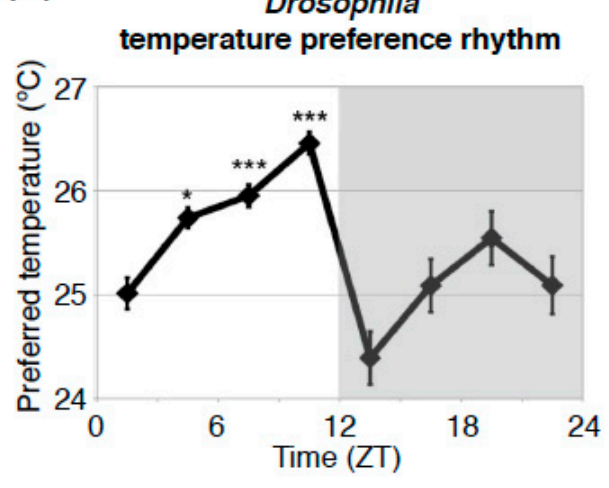

Figure 1. Comparison between the human body temperature rhythm (BTR) and the Drosophila temperature preference rhythm (TPR). The human BTR (A) (replotted the figure using an example form Duffy JF et al. 1998, Figure 3 [3]) and the Drosophila TPR (B) (modified from Kaneko et al. 2012, Figure 1 [18]). The shadow area in (A) shows the sleep period, and (B) shows the dark period. ZT: zeitgeber time. ${ }^{*}: p<0.05,{ }^{* * *}: p<0.001$ (comparison to ZT 1-3). Given that the body temperature of Drosophila is close to their ambient temperature, the TPR results in fluctuations in body temperature. The TPR shows a rhythmic pattern similar to that of the human BTR.

\section{Drosophila Exhibit a Robust Temperature Preference Rhythm (TPR)}

To determine the molecular and neural mechanisms by which the circadian clock regulates BTRs, we first designed a behavioral experiment to monitor the BTR in Drosophila. Because Drosophila are small ectotherms, their body temperatures are very close to the temperature of their surroundings [7,14-17]. Therefore, we sought to monitor their surrounding temperatures and conduct a behavioral assay of temperature preference (Figure 2) [24-26]. Drosophila exhibit robust temperature preference behavior in 12:12-h light-dark (LD) cycles; they avoid warm and cold temperatures and prefer $\sim 25^{\circ} \mathrm{C}$ in the morning, which suggests that the flies' body temperatures are very close to $\sim 25^{\circ} \mathrm{C}$ at that time [25]. We monitored their preferred temperatures for $24 \mathrm{~h}$ and found that the preferred temperatures fluctuated over that period; the preferred temperature was $25^{\circ} \mathrm{C}$ in the morning, then increased during the daytime and decreased during the night (Figure 1B) [18]. We refer to this rhythmic change in preferred temperature as temperature preference rhythm (TPR). Given that the body temperature in Drosophila is close to their ambient temperature, the TPR results in a Drosophila BTR. Importantly, the Drosophila TPR shows a rhythmic pattern similar to that of the human BTR [3] (Figure 1). Furthermore, we demonstrated that TPR is a clock-controlled behavior based on the following observations: we showed that the fluctuation of preferred temperature was still maintained in constant darkness [27] and that two clock mutants—imeless and period mutant flies (tim ${ }^{01}$ and per $^{01}$, respectively)—lost TPR in both LD and 
DD [18]. The data suggest that TPR is not solely generated by a response to light inputs but is regulated by an endogenous circadian clock. Thus, we discovered that Drosophila exhibit a robust TPR, which generates a BTR in flies.

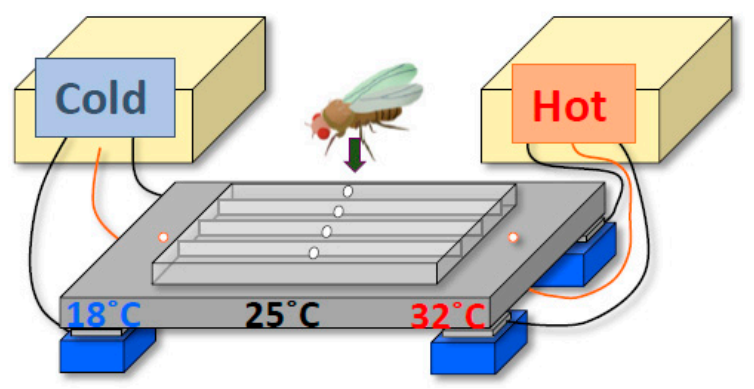

Figure 2. Drosophila temperature preference behavioral assay. A temperature gradient from 18 to $32{ }^{\circ} \mathrm{C}$ is generated in a chamber made with a metal plate and a plexiglass cover. Flies are introduced into the chamber through the holes in the cover. Within 30 minutes, the flies settle in the locations with their preferred temperatures. Because their body temperatures are very close to the ambient temperature, their body temperatures can be determined by measuring the temperature in the place where they are located. The diagram is modified from Goda et al. 2014, Figure 3 [26].

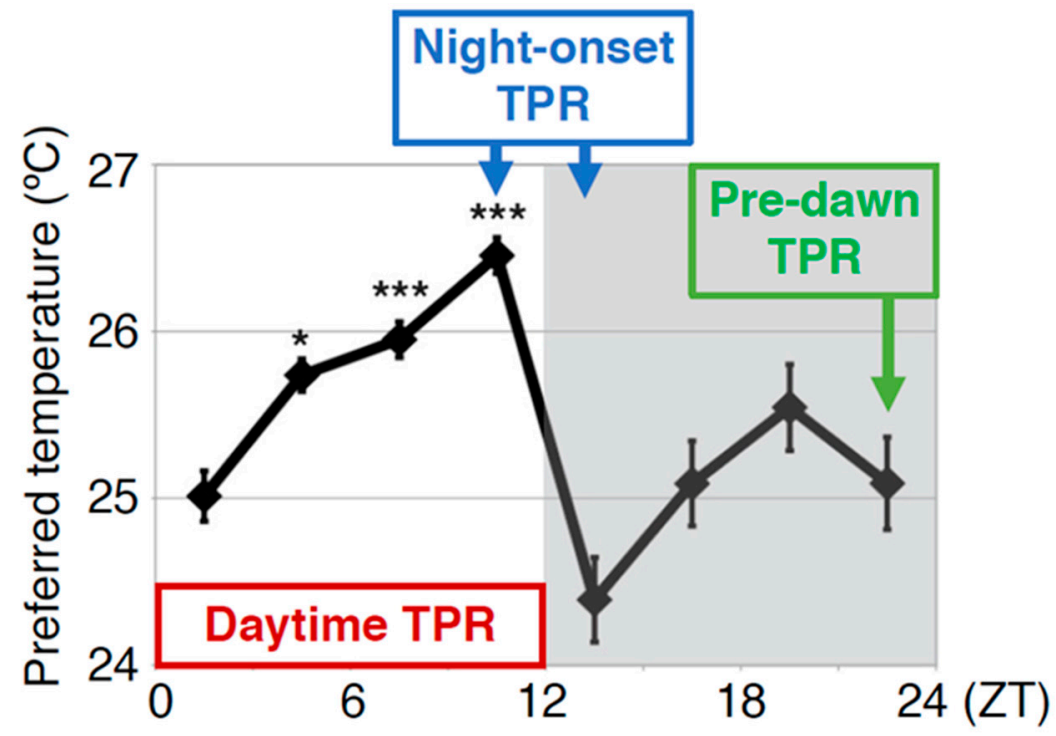

Figure 3. The TPR is regulated by different regulatory mechanisms at different times of the day: daytime, night onset, and predawn. Daytime TPR: The increase in preferred temperature during the daytime (ZT 1-12: shown by the red rectangle). Night-onset TPR: The dramatic decrease in preferred temperature at the transition from day to night (ZT 10-15: shown by blue rectangle). Predawn TPR: The preferred temperature just before dawn is similar to that of early morning (ZT 22-24: shown by green rectangle). The TPR is regulated by different regulatory mechanisms in each of these periods. The graph is modified from Kaneko et al. 2012, Figure 1 [18]. *: $p<0.05,{ }^{* * *}: p<0.001$ (comparison to ZT 1-3).

\section{TPR is Controlled by the Noncanonical Clock Circuits via Dorsal Neurons 2 (DN2s)}

Because several researches in humans and rats suggest that BTR is controlled separately from locomotor activity rhythms [7,19-22], we asked whether Drosophila TPR is also controlled separately from locomotor activity rhythms. There are 150 central pacemaker neurons in the fly brain, and they have functions similar to those of mammalian suprachiasmatic nucleus (SCN) neurons [28]. Based on their locations in the brain, pacemaker neurons are divided into four groups of lateral neurons (small ventrolateral neurons (sLNvs), large LNvs (lLNvs), dorsolateral neurons (LNds), and lateral posterior 
neurons (LPNs)) and three groups of dorsal neurons (DN1s, DN2s, and DN3s) [29]. We demonstrated that DN2s are the main clock neurons that regulate TPR, but DN2s do not regulate locomotor activity rhythms [18]. Thus, TPRs and locomotor activity rhythms are regulated by distinct pathways. Interestingly, the TPR persists when constant light (LL) is maintained for eight days, whereas locomotor activity rhythms are disrupted by four days of LL conditions [18]. This result also implies that TPR and locomotor activity rhythms are separately regulated.

\section{Molecular and Neural Mechanisms Regulating TPR}

To understand the molecular mechanisms of TPR, we focused on neuropeptides and receptors because they control many behaviors and physiological activities. In this review, we summarize the clock neurons and neuropeptide signaling that control the TPR at different times of the day: daytime (zeitgeber time (ZT) 1-12), night onset (ZT 10-15) and before dawn (ZT 22-24) (Figure 3).

\subsection{Diuretic Hormone 31 Receptor (DH31R) Regulates the Daytime TPR (ZT 1-12)}

The neuropeptide pigment-dispersing factor (PDF) and its receptor (PDFR) play important roles in the synchronization of molecular rhythms among core clock neurons and maintaining robust locomotor activity rhythms in Drosophila [30-35]. Therefore, we initially anticipated the involvement of PDF-PDFR signaling in TPR. However, both $P d f$ mutant $\left(P d f^{01}\right)$ and $P d f r$ mutant $\left(P d f r^{5304}\right)$ flies exhibited a normal increase in preferred temperatures during the daytime [23], suggesting that PDF and PDFR do not have a main role in regulating the daytime TPR.

DH31R belongs to the class B G-protein coupled receptor (GPCR) family, the same family as PDFR [36]. The ligand DH31 can activate DH31R as well as PDFR [34,37,38], and DH31 is also involved in sleep before dawn [39]. Therefore, we next asked whether DH31R plays an important role in the TPR. We found that $D h 31 r$ mutant $\left(D h 31 r^{1 / D f}\right)$ flies exhibited a constant preferred temperature of approximately $27^{\circ} \mathrm{C}$ during the daytime (Figure 4) [23]. The Dh31r mutant also disrupted the daytime TPR in DD, suggesting that the function of DH31R in the daytime TPR is associated with the endogenous circadian clock.

(A)

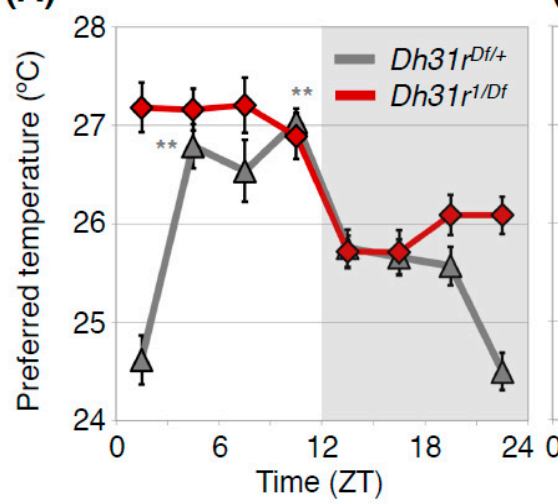

(B)

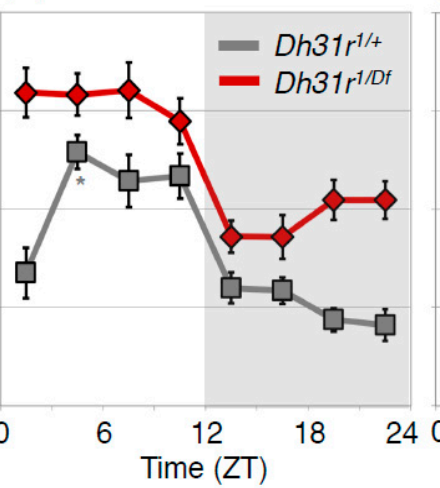

(C)

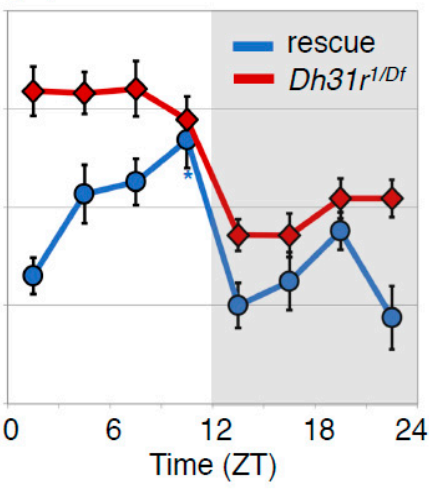

Figure 4. DH31R regulates the daytime TPR. Comparison of TPR between Dh31r mutant flies (Dh31r1/Df , red line in $(\mathbf{A}-\mathbf{C})$ and heterozygous control flies $\left(D h 31 r^{1 /+}\right.$, gray line in $(\mathbf{A})$ or $D h 31 r^{D f /+}$, gray line in (B)) or Dh31r genomic rescue flies (rescue, blue line in (C)). Dh31r mutant flies show an abnormal daytime TPR, in which they prefer a constant temperature of approximately $27^{\circ} \mathrm{C}$ during the daytime. The control flies exhibit a normal daytime TPR, in which their preferred temperature increases during the daytime (A,B). Genomic rescue of $D h 31 r$ flies restored normal daytime TPR (C). Dh31r ${ }^{1}$ is a P-element insertion mutant (PBac $\left.\{W H\} D h 31-R^{f 05546}\right)$, and $D h 31 r^{D f}$ is a deletion mutant [Df(2R) BSC273]. In the Dh31r genomic rescue fly, the Ch321-57F06 BAC clone, which includes the entire Dh31r gene region, is inserted into the genome. The graphs are modified from Goda et al. 2018, Figure 1 [23]. *: $p<0.05,{ }^{* *}: p<0.01$ (comparison to ZT 1-3). 
Because DN2s are the main clock neurons for the TPR [18], we expected that DH31R might be expressed in DN2s. However, immunostaining with an anti-DH31R antibody revealed that DH31R is not expressed in DN2s but is expressed in a subset of DN1s, DN3s, and lLNvs [23]. To determine whether DH31R expression in these clock neurons is important for the TPR, we performed rescue experiments and found that Dh31r expression in all clock neurons restored the TPR. This result suggests that DH31R in clock cells is sufficient for regulating the daytime TPR (Figure 7A).

How does DH31R in clock cells regulate the daytime TPR? We recognized two possibilities, as follows: (1) DH31R controls the molecular oscillations of clock genes; or (2) DH31R acts on the downstream output of clock neurons. To examine these possibilities, we monitored the molecular oscillations in core clock neurons, including DN2s, and found that they were intact in Dh31r mutant flies. These data suggest that DH31R regulates the daytime TPR by acting downstream of clocks [23]. Notably, we also demonstrated that Dh31r mutant flies exhibited normal locomotor activity rhythms [23]. These data provide further support for the idea that TPR and locomotor activity rhythms are regulated separately.

\subsection{Both DH31 and PDF Contribute to the Regulation of the Daytime TPR (ZT 1-12)}

Next, we asked whether mutants of Dh31, a ligand of DH31R, showed an abnormal daytime TPR. However, Dh31, as well as Pdf mutants, showed a normal daytime TPR [40]. Given that both Dh31 and Pdf single mutants showed a normal daytime TPR, we hypothesized that DH31 and PDF might have a redundant function. To examine this possibility, we created Dh31 and Pdf double mutant flies and found that they had a disrupted daytime TPR, with a constant preferred temperature during the daytime [23]. To determine whether DH31 and PDF act on the clock neurons, we performed rescue experiments using membrane-tethered peptides, which localize on the cell surface and prevent spreading [41]. We demonstrated that abnormal TPR in Dh31 and Pdf double mutant flies were recovered by the expression of either tethered-Dh31 (t-Dh31) or tethered-Pdf (t-Pdf) in all clock cells [23]. These data suggest that both DH31 and PDF act on the clock cells to regulate the daytime TPR. It would be of interest to further investigate the relationships between DH31, PDF, and DH31R in terms of the regulation of the TPR.

\subsection{DH31 Acts on DN2s Through PDFR, but not DH31R, to Regulate the Night-onset TPR (ZT 10-15)}

The preferred temperature for Drosophila drops dramatically by approximately $1.5^{\circ} \mathrm{C}$ at the transition from day to night (night-onset TPR) (Figure 3). In Dh31 mutant flies, the dramatic decrease in preferred temperature at the transition from day to night was dampened to $0.6 \sim 0.8{ }^{\circ} \mathrm{C}$ (Figure 5D) [40], suggesting that DH31 is responsible for the night-onset TPR. To identify the neurons that regulate the night-onset TPR through DH31, we expressed t-DH31 in a subset of clock cells in Dh31 mutant flies. We demonstrated that $\mathrm{t}$-DH31 expression in DN2s rescued the decrease in preferred temperature at the transition from day to night in Dh31 mutant flies, suggesting that DH31 signaling acts on DN2s to regulate the night-onset TPR.

What is the receptor of DH31 for the regulation of the night-onset TPR? We found that Pdfr mutant flies exhibited a dampened night-onset TPR (Figure 5B) [40]. Because it has been shown that DH31 can activate PDFR $[34,37,38]$, we speculated that DH31 activates PDFR to regulate the night-onset TPR. To test this possibility, we expressed PDFR in a subset of clock cells, including DN2s, in Pdfr mutant flies. We found that PDFR expression in DN2s restored a robust night-onset TPR, indicating that PDFR in DN2s is sufficient for the night-onset TPR. Taken together, these data suggest that DH31 acts on DN2s through PDFR to regulate the night-onset TPR (Figure 7B). Importantly, although DH31 activates DH31R, the Dh31r mutants exhibit a normal night-onset TPR. Because DH31R is required for the daytime TPR but not for the night-onset TPR, it appears that the daytime and night-onset TPRs are regulated by different mechanisms. 
(A) daytime

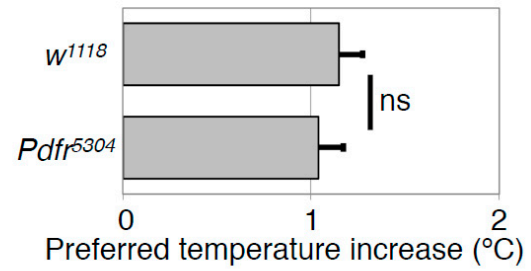

(B) night-onset

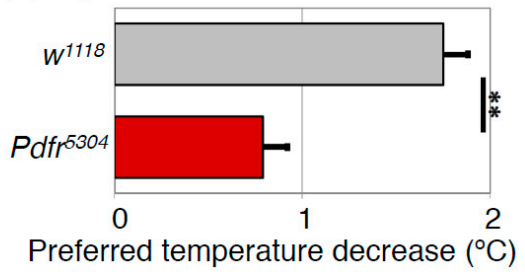

(C) daytime

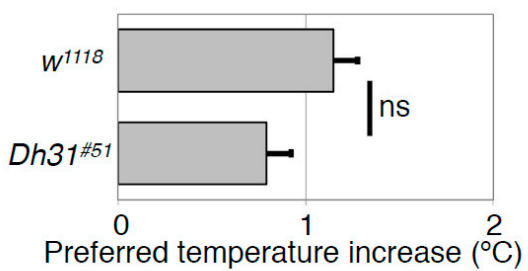

(D) night-onset

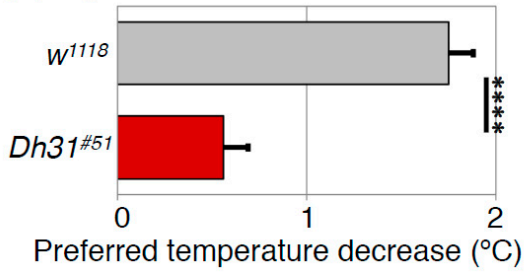

Figure 5. PDFR and DH31 regulate the night-onset TPR. Comparison of daytime TPR $(\mathrm{A}, \mathrm{C})$ or night-onset TPR (B,D) between $w^{1118}$ and Pdfr mutant $\left(P d f r^{5304}\right)(\mathbf{A , B})$ or Dh31 mutant $\left(D h 31^{\# 51}\right)(\mathbf{C}, \mathbf{D})$ flies. Both Pdfr ${ }^{5304}$ and $D h 31^{\# 51}$ flies show significantly dampened night-onset TPR (B,D), while both exhibit robust increases in preferred temperature during the daytime $(\mathbf{A}, \mathbf{C})$. The data suggest that both PDFR and DH31 are responsible for the night-onset TPR. The graphs are modified from Goda et al. 2016, Figures 1 and 3 [40]. t-test: ${ }^{* *}: p<0.01,{ }^{* * * *}: p<0.0001$.

\subsection{PDF Neurons Regulate the Predawn TPR via DN2s (ZT 22-24)}

In the wild type flies, the preferred temperature before dawn (ZT22-24) is similar to that of early morning at ZT1-3 (predawn TPR). To understand the mechanisms of the predawn TPR, we first focused on the PDF neurons because they regulate morning anticipation in locomotor activity rhythms; Pdf mutant flies show a lack of morning anticipation, a phenomenon that is manifested as an increase in locomotor activity before dawn [42]. Therefore, we asked whether PDF is involved in regulating TPR before dawn. We found that Pdf mutant flies preferred a lower nighttime temperature (ZT 16-24) than wild-type flies (Figure 6) [43]. A similar phenotype was observed in the flies whose PDF neurons were inhibited by expressing the mammalian inward rectifier $\mathrm{K}^{+}$channel Kir2.1 [44]. These data suggest that PDF neurons play an important role in regulating the predawn TPR.

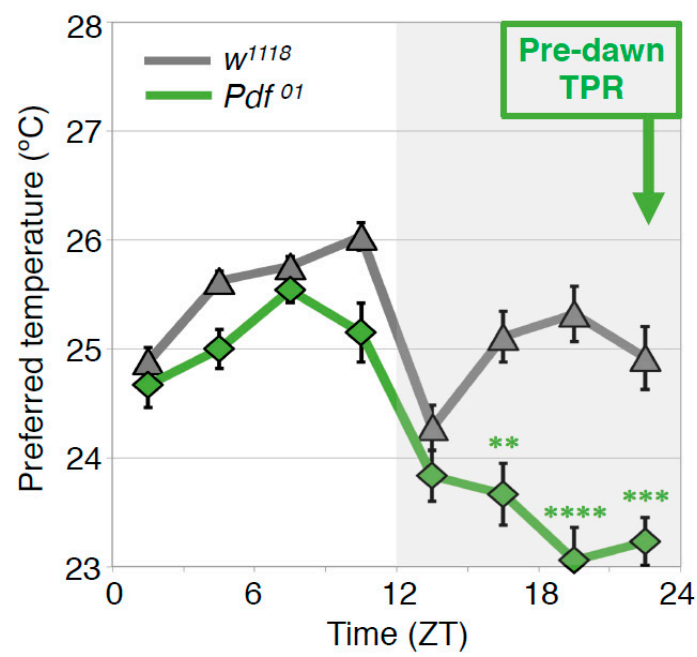

Figure 6. PDF regulates the predawn TPR. Comparison of TPRs between $w^{1118}$ and Pdf mutant $\left(P d f^{01}\right)$ flies. $P d f^{01}$ flies exhibit robust daytime (ZT 1-12) and night-onset TPRs (ZT 10-15). However, their preferred temperatures continue decreasing during the night and are much lower before dawn (ZT 22-24) than that of $w^{1118}$ flies (predawn TPR). These data suggest that PDF is important for the predawn TPR. t-test between $w^{1118}$ and Pdf ${ }^{01}$ in each ZT: ${ }^{* *}: p<0.01{ }^{* * * *}: p<0.001,{ }^{* * * *}: p<0.0001$. 
Because PDF neurons are not the main pacemaker neurons for the TPR [18], we wondered what roles PDF neurons might play in regulating the predawn TPR. Because sLNvs, a subset of PDF neurons, are known to contact DN2s in larvae [45] and project near DN2s in adult, the main pacemaker neurons for the TPR [46], we hypothesized that sLNvs might contact DN2s to regulate the predawn TPR. Using green fluorescent protein (GFP) reconstitution across synaptic partners (GRASP) analysis [47-49], we showed that sLNvs make contacts with DN2s and that the number of contacts fluctuates over $24 \mathrm{~h}$, with a peak before dawn [43].

Using a genetically encoded calcium indicator, GCaMP with the adenosine triphosphate (ATP)-gated ionotropic purinoreceptor, P2X2 [50], we showed that sLNvs functionally contact and activate DN2s [43]. The data suggest that sLNvs and DN2s have a temporal interaction and that sLNvs manipulate the activity of DN2s. However, the role of DN2s other than as the main clock remained unclear. Therefore, we examined how DN2 activity affects the TPR. We tested the TPR in flies whose DN2s were inhibited by Kir2.1 [44]. Our data showed that the inhibition of DN2s caused a reduced preferred temperature at all times in the circadian cycle compared to that of the control flies [43]. Thus, it is likely that sLNvs activate DN2s to increase the preferred temperature at dawn. Together, our data suggest that the temporal interaction between sLNvs and DN2s peaks before dawn and that sLNv-DN2 neural circuits regulate the setpoint of preferred temperature before dawn (Figure 7C).

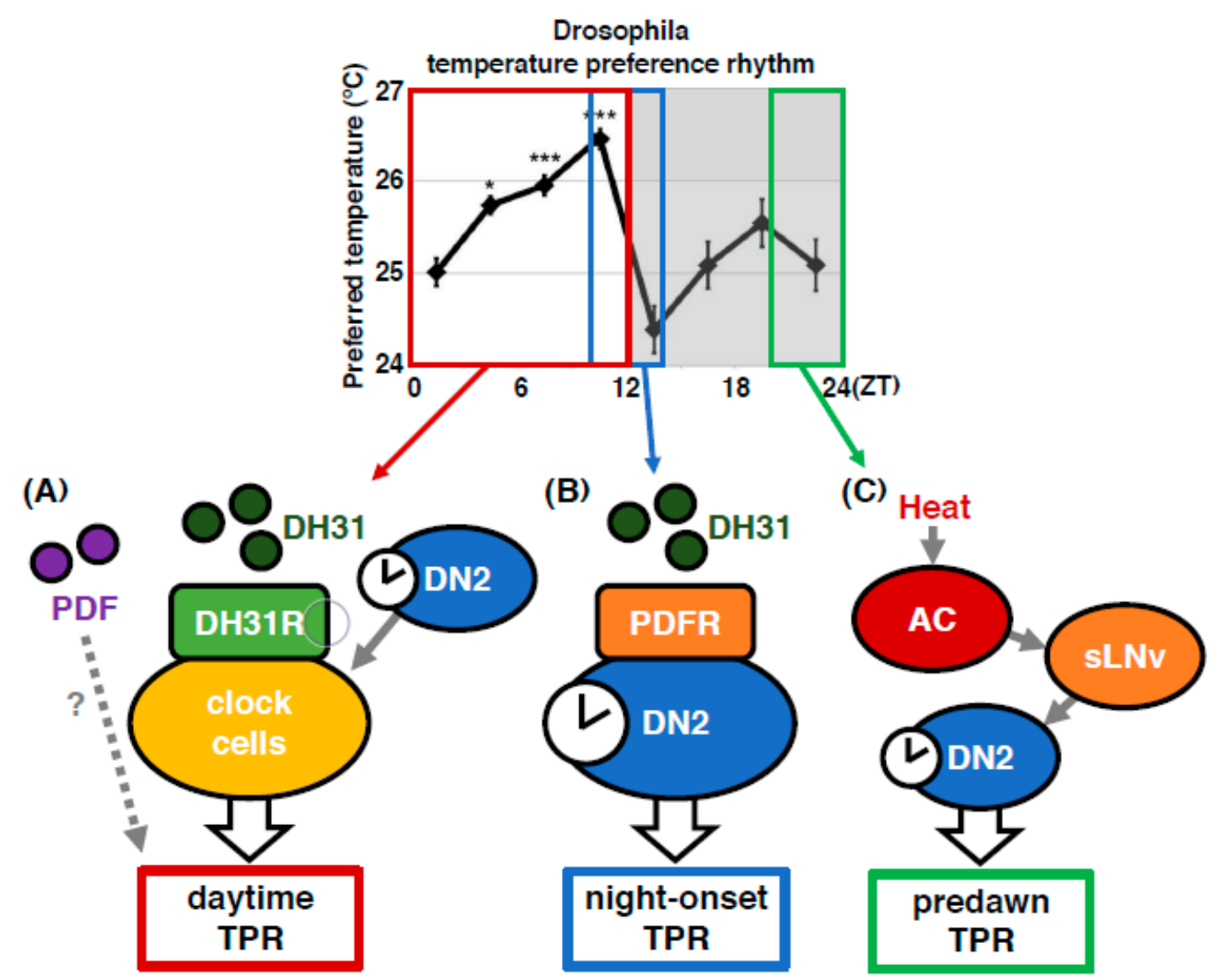

Figure 7. The Drosophila TPR is regulated by different mechanisms at different times of the day. Models for regulatory mechanisms of the TPR in each time range: daytime (ZT 1-12), night onset (ZT 10-15), and predawn (ZT 22-24). The daytime TPR: DN2s are the main clock neurons. DH31 acts on DH31R in a subset of clock neurons to regulate the daytime TPR. PDF is also involved in daytime TPR regulation. The night-onset TPR: DH31 acts on DN2s via PDFR to regulate the night-onset TPR. The predawn TPR: An AC-sLN-DN2 neural circuit regulates the proper setting of temperature preference before dawn. 


\subsection{Anterior Cell (AC) Neurons Regulate the Predawn TPR via sLNvs (ZT 22-24)}

How are the clock neurons interacted with the temperature-sensing neurons? One of the Drosophila transient receptor potential A (TRPA) superfamilies, TRPA1, which is activated above $25^{\circ} \mathrm{C}$, is expressed in anterior cell (AC) neurons located in the brain along with the antennal nerve [25,51]. TRPA1 in AC neurons is responsible for temperature preference behavior [25].

Because both AC neurons and sLNvs project to the dorsal protocerebrum [25,46,52,53], we asked whether sLNvs interact with AC neurons. Using GRASP, we demonstrated that sLNvs have contacts with AC neurons [43]. To determine whether sLNv-AC circuits are the important neural circuits for the predawn TPR, we asked whether AC inhibition causes a lower preferred temperature phenotype similar to that of sLNv-inhibited flies. We demonstrated that either TrpA1 knockdown in AC neurons or inhibition of AC neurons by Kir2.1 resulted in a lower preferred temperature from late night until just before dawn (ZT 19-24), which is a phenotype similar to the one resulting from Pdf mutation or inhibition of PDF neurons. The data suggest that AC neurons regulate the temperature setpoint before dawn and that sLNv-AC circuits are the important neural circuits for the predawn TPR. Given that AC neurons are serotonergic [52] and LNvs express one of the serotonin receptors, 5-hydroxytryptamine receptor 1B, 5HT1B [54], we asked whether serotoninergic transmission from AC neurons to sLNvs contributes to the regulation of the predawn TPR. We found that knocking down 5HT1B in sLNvs caused a reduced preferred temperature before dawn, which is similar to the phenotype observed in AC- or sLNv-inhibited flies. Therefore, our data suggest that the sLNv-AC neural circuits regulate the predawn TPR via serotonin signaling.

\section{Mammalian BTRs}

Human body temperatures increase gradually during wakefulness and decrease during sleep (Figure 1A) [3-6]. BTR is controlled by the circadian clock, balancing heat production and heat loss for $24 \mathrm{~h}$ via physiological and behavioral approaches $[55,56]$. As daily variations in BTR are robust and parallel fluctuations in locomotor activity rhythms, BTR is widely used to monitor circadian rhythms in mammals. The molecular mechanisms that regulate BTR remain largely uncharacterized, although a study in which subsets of neurons in rats were surgically ablated suggested that locomotor activity rhythms and BTRs are controlled by different output pathways that originate from the suprachiasmatic nucleus (SCN) [19]. In humans, body temperature fluctuates even when locomotor activity is restricted $[20,21]$, and BTR and locomotor activity rhythms can be experimentally dissociated, a phenomenon known as spontaneous internal desynchronization [22,57]. These data suggest that the BTR is controlled in a manner distinct from that of locomotor activity rhythms. However, molecular evidence supporting this possibility has not been reported. Therefore, there was-and remains-a critical need to identify mechanisms that regulate BTRs.

\section{The Calcitonin Receptor Regulates the BTR in Mice}

Given that many fundamental biological mechanisms are functionally conserved between flies and mammals, we asked whether a mammalian homolog of DH31R was also involved in the regulation of mammalian BTRs. The closest mammalian homologs of Drosophila DH31R are the calcitonin receptor (Calcr) and the calcitonin receptor-like receptor (Calcrl) [36]. Both Calcr and Calcrl are seven-transmembrane-domain class B GPCRs and are involved in physiological functions including calcium metabolism [58-60]. The amino acid sequence similarity between DH31R and Calcr or Calcrl is $67.9 \%$ or $67.4 \%$, respectively, in the seven transmembrane domains and $74.6 \%$ or $81.0 \%$, respectively, in the N-terminal region [23]. Because Calcr is expressed in the SCN in rats and mice, whereas Calcrl is not [61-63], we focused on Calcr and examined its role in the BTR using mice.

To determine where Calcr is expressed in the SCN, we performed in situ hybridization as well as immunohistochemistry using an anti-Calcr antibody. We demonstrated that Calcr mRNA and Calcr protein were similarly distributed in the dorsomedial area of the $\mathrm{SCN}$, a region corresponding to the 
SCN shell. Arginine vasopressin (AVP) and vasoactive intestinal polypeptide (VIP) are markers of the SCN shell and SCN core, respectively [64]. We performed double immunostaining with anti-Calcr and anti-AVP or anti-VIP antibodies and found that AVP and Calcr partially overlapped in the SCN shell, whereas VIP and Calcr did not colocalize. These data also suggest that Calcr is expressed in the SCN shell but not in the VIPergic SCN [23].

To examine whether Calcr plays an important role in the circadian rhythm of body temperature, we compared the BTRs of wild-type and Calcr knockout (KO) mice [23]. The body temperature of wild-type mice fluctuates over a 24-h period [3]. Because mice are nocturnal animals, their BTR patterns are different from those of humans. During the daytime, when mice are primarily resting, their body temperature gradually decreases during the early phase and increases during the late phase [65-67]. However, during the night (the active phase of mice), the animals' body temperature displays bimodal peaks in the early night and at dawn, with a deep trough late at night (i.e., the midnight trough) $[67,68]$. Although we found that the body temperatures of both wild-type and Calcr KO mice fluctuated over a 24-h period, they were significantly different at midnight. Specifically, the body temperatures of wild-type mice showed a deep trough at midnight, whereas the body temperatures of Calcr KO mice lost the characteristic trough and remained relatively unchanged during the night (Figure 8A). These data indicate that the lack of Calcr expression causes a shallow midnight trough in body temperature, suggesting that Calcr is required for body temperature fluctuations, particularly during the night (the active phase of mice). Importantly, this finding is consistent with the findings for the TPR phenotype of the Drosophila Dh31r mutant, as we observed that the Dh31r mutation caused a flat TPR during the daytime (the active phase of flies). Therefore, both Drosophila DH31R and mouse Calcr are required for body temperature fluctuation during the active phase [23].

(A)

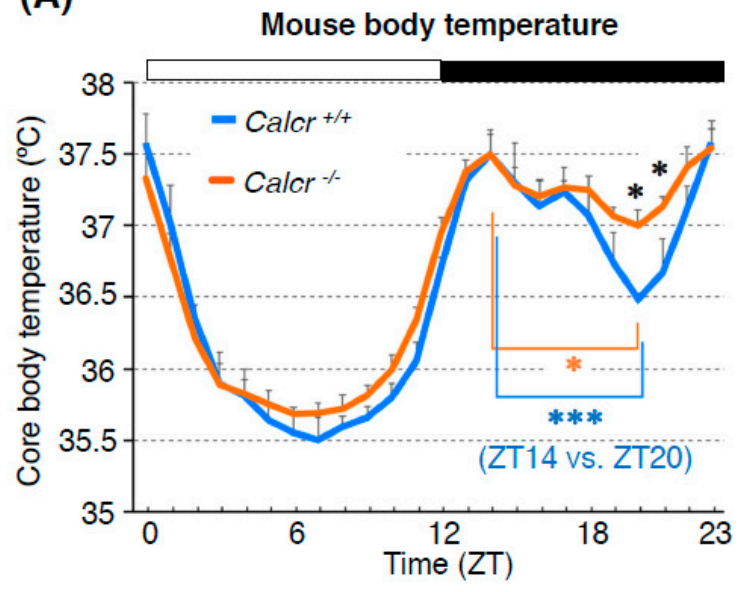

(B)

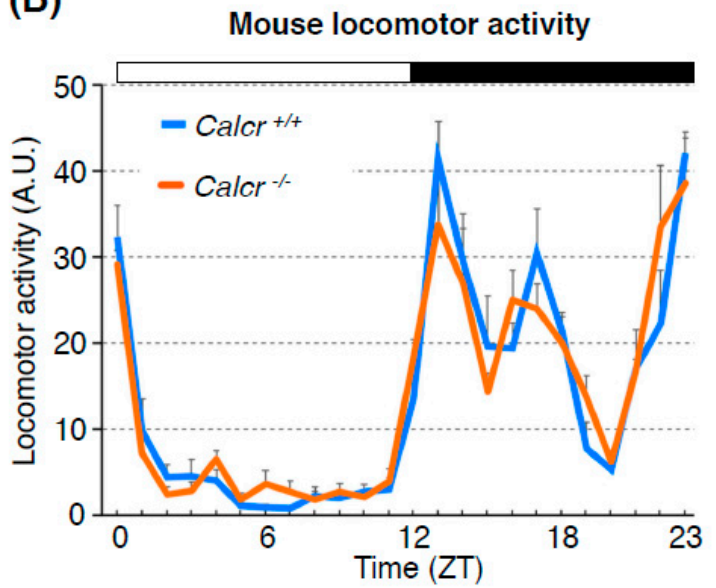

Figure 8. Calcr contributes to body temperature regulation during the night (the active phase for mice). Mouse BTR (A) and locomotor activity rhythm (B) in control ( $\mathrm{Calcr}^{+/+}$: blue line) and Calcr knockout ( $\mathrm{Calcr}^{-/-}$: orange line) mice. The body temperatures of wild-type mice showed a deep trough at midnight, whereas the body temperatures of Calcr knockout mice lost the characteristic trough and remained relatively unchanged during the night (A). The locomotor activity rhythm in Calcr knockout did not show a significant difference from that of the control, suggesting that Calcr is responsible for mammalian body temperature regulation but not locomotor activity rhythm during the night (the active phase for mice). White and black bars on the graphs indicate the 12-h light and dark phases. The graphs are modified from Goda et al. 2018, Figure 6 [23]. *: $p<0.05,{ }^{* * *}: p<0.001$.

Because body temperature may be increased by locomotor activity, it is possible that increased levels of locomotor activity result in the elevated body temperatures observed in Calcr KO mice. To examine this possibility, we compared fluctuations in locomotor activity between wild-type and Calcr KO mice; however, there was no significant difference in locomotor activity between the two 
groups (Figure 8B). Therefore, we concluded that Calcr specifically mediates body temperature fluctuations during the night and does not affect locomotor activity rhythms.

Together, our data demonstrated that DH31R mediates the Drosophila TPR and that calcitonin Calcr is essential for the normal BTR in mice [23]. Importantly, neither DH31R nor Calcr regulates locomotor activity rhythms. Although the mechanisms that underlie thermoregulation in Drosophila and mammals are completely different, our data identify the calcitonin receptors DH31R and Calcr as fundamental, ancient mediators of daily BTRs in both flies and mice. Thus, the Drosophila TPR is a functionally conserved model for mammalian BTRs, and our study using the Drosophila TPR will provide fundamental insights into the molecular and neural mechanisms that control BTRs in mammals.

\section{Conclusions}

BTRs are essential for homeostatic functions and are regulated separately from locomotor activity rhythms [7]. Our studies over the past several years established that the molecular and neural mechanisms that control the fly TPR resemble those of the mammalian BTR $[18,26,40,43]$. We also determined that calcitonin receptors (fly DH31R and mouse Calcr) are ancient regulators of the fly TPR and the mouse BTR [23]. The powerful genetic tools available in flies will allow us to reveal mechanisms that have remained a long-standing mystery: the mechanisms that control how body temperature increases during wakefulness and decreases during sleep. Thus, our expectation is that the fly TPR will serve as an innovative model to understand the mammalian BTR. The outcome of this research is expected to establish important foundations for our understanding of the daily rhythms of body temperature in both flies and mammals, bearing important implications for the treatment of circadian clock diseases, sleep problems, metabolic diseases, and the health of night-shift workers.

Author Contributions: F.N.H. and T.G. wrote the manuscript.

Funding: This review was supported by NIH R01 grant GM107582 to F.N.H.

Acknowledgments: We thank the members of the Hamada laboratory for their critical comments and advice on the manuscript.

Conflicts of Interest: The authors declare no conflict of interest.

\section{References}

1. Partch, C.L.; Green, C.B.; Takahashi, J.S. Molecular architecture of the mammalian circadian clock. Trends Cell Biol. 2014, 24, 90-99. [CrossRef] [PubMed]

2. Dubowy, C.; Sehgal, A. Circadian Rhythms and Sleep in Drosophila melanogaster. Genetics 2017, 205, 1373-1397. [CrossRef] [PubMed]

3. Duffy, J.F.; Dijk, D.J.; Klerman, E.B.; Czeisler, C.A. Later endogenous circadian temperature nadir relative to an earlier wake time in older people. Am. J. Physiol. 1998, 275, R1478-R1487. [CrossRef]

4. Aschoff, J. Circadian Control of Body-Temperature. J. Therm. Biol. 1983, 8, 143-147. [CrossRef]

5. Krauchi, K. How is the circadian rhythm of core body temperature regulated? Clin. Auton. Res. 2002, 12, 147-149. [CrossRef]

6. Weinert, D. Circadian temperature variation and ageing. Ageing Res. Rev. 2010, 9, 51-60. [CrossRef] [PubMed]

7. Refinetti, R.; Menaker, M. The circadian rhythm of body temperature. Physiol. Behav. 1992, 51, $613-637$. [CrossRef]

8. Gilbert, S.S.; van den Heuvel, C.J.; Ferguson, S.A.; Dawson, D. Thermoregulation as a sleep signalling system. Sleep Med. Rev. 2004, 8, 81-93. [CrossRef]

9. Krauchi, K. The thermophysiological cascade leading to sleep initiation in relation to phase of entrainment. Sleep Med. Rev. 2007, 11, 439-451. [CrossRef] [PubMed]

10. Krauchi, K. The human sleep-wake cycle reconsidered from a thermoregulatory point of view. Physiol. Behav. 2007, 90, 236-245. [CrossRef]

11. Buhr, E.D.; Yoo, S.H.; Takahashi, J.S. Temperature as a universal resetting cue for mammalian circadian oscillators. Science 2010, 330, 379-385. [CrossRef] 
12. Morf, J.; Schibler, U. Body temperature cycles: Gatekeepers of circadian clocks. Cell Cycle 2013, 12, 539-540. [CrossRef]

13. Brown, S.A.; Zumbrunn, G.; Fleury-Olela, F.; Preitner, N.; Schibler, U. Rhythms of mammalian body temperature can sustain peripheral circadian clocks. Curr. Biol. 2002, 12, 1574-1583. [CrossRef]

14. Stevenson, R.D. The Relative Importance of Behavioral and Physiological Adjustments Controlling Body-Temperature in Terrestrial Ectotherms. Am. Nat. 1985, 126, 362-386. [CrossRef]

15. Ellis, D.J.; Firth, B.T.; Belan, I. Circadian rhythms of locomotor activity and temperature selection in sleepy lizards, Tiliqua rugosa. J. Comp. Physiol. A Neuroethol. Sens. Neural Behav. Physiol. 2007, 193, 695-701. [CrossRef] [PubMed]

16. Dillon, M.E.; Wang, G.; Garrity, P.A.; Huey, R.B. Review: Thermal preference in Drosophila. J. Therm. Biol. 2009, 34, 109-119. [CrossRef] [PubMed]

17. Garrity, P.A.; Goodman, M.B.; Samuel, A.D.; Sengupta, P. Running hot and cold: Behavioral strategies, neural circuits, and the molecular machinery for thermotaxis in C. elegans and Drosophila. Genes Dev. 2010, 24, 2365-2382. [CrossRef]

18. Kaneko, H.; Head, L.M.; Ling, J.; Tang, X.; Liu, Y.; Hardin, P.E.; Emery, P.; Hamada, F.N. Circadian rhythm of temperature preference and its neural control in Drosophila. Curr. Biol. 2012, 22, 1851-1857. [CrossRef] [PubMed]

19. Saper, C.B.; Lu, J.; Chou, T.C.; Gooley, J. The hypothalamic integrator for circadian rhythms. Trends Neurosci. 2005, 28, 152-157. [CrossRef] [PubMed]

20. Smith, R.E. Circadian variations in human thermoregulatory responses. J. Appl. Physiol. 1969, 26, 554-560. [CrossRef]

21. Gander, P.H.; Connell, L.J.; Graeber, R.C. Masking of the circadian rhythms of heart rate and core temperature by the rest-activity cycle in man. J. Biol. Rhythms 1986, 1, 119-135. [CrossRef]

22. Lavie, P. Sleep-wake as a biological rhythm. Annu. Rev. Psychol. 2001, 52, 277-303. [CrossRef]

23. Goda, T.; Doi, M.; Umezaki, Y.; Murai, I.; Shimatani, H.; Chu, M.L.; Nguyen, V.H.; Okamura, H.; Hamada, F.N. Calcitonin receptors are ancient modulators for rhythms of preferential temperature in insects and body temperature in mammals. Genes Dev. 2018, 32, 140-155. [CrossRef]

24. Sayeed, O.; Benzer, S. Behavioral genetics of thermosensation and hygrosensation in Drosophila. Proc. Natl. Acad. Sci. USA 1996, 93, 6079-6084. [CrossRef]

25. Hamada, F.N.; Rosenzweig, M.; Kang, K.; Pulver, S.R.; Ghezzi, A.; Jegla, T.J.; Garrity, P.A. An internal thermal sensor controlling temperature preference in Drosophila. Nature 2008, 454, 217-220. [CrossRef]

26. Goda, T.; Leslie, J.R.; Hamada, F.N. Design and analysis of temperature preference behavior and its circadian rhythm in Drosophila. J. Vis. Exp. 2014, e51097. [CrossRef]

27. Morrison, S.F.; Nakamura, K.; Madden, C.J. Central control of thermogenesis in mammals. Exp. Physiol. 2008, 93, 773-797. [CrossRef]

28. Welsh, D.K.; Takahashi, J.S.; Kay, S.A. Suprachiasmatic nucleus: Cell autonomy and network properties. Annu. Rev. Physiol. 2010, 72, 551-577. [CrossRef]

29. Dubruille, R.; Emery, P. A plastic clock: How circadian rhythms respond to environmental cues in Drosophila. Mol. Neurobiol. 2008, 38, 129-145. [CrossRef]

30. Renn, S.C.; Park, J.H.; Rosbash, M.; Hall, J.C.; Taghert, P.H. A pdf neuropeptide gene mutation and ablation of PDF neurons each cause severe abnormalities of behavioral circadian rhythms in Drosophila. Cell 1999, 99, 791-802. [CrossRef]

31. Lin, Y.; Stormo, G.D.; Taghert, P.H. The neuropeptide pigment-dispersing factor coordinates pacemaker interactions in the Drosophila circadian system. J. Neurosci. 2004, 24, 7951-7957. [CrossRef]

32. Lear, B.C.; Zhang, L.; Allada, R. The neuropeptide PDF acts directly on evening pacemaker neurons to regulate multiple features of circadian behavior. PLoS Biol. 2009, 7, e1000154. [CrossRef] [PubMed]

33. Hyun, S.; Lee, Y.; Hong, S.T.; Bang, S.; Paik, D.; Kang, J.; Shin, J.; Lee, J.; Jeon, K.; Hwang, S.; et al. Drosophila GPCR Han is a receptor for the circadian clock neuropeptide PDF. Neuron 2005, 48, 267-278. [CrossRef] [PubMed]

34. Mertens, I.; Vandingenen, A.; Johnson, E.C.; Shafer, O.T.; Li, W.; Trigg, J.S.; De Loof, A.; Schoofs, L.; Taghert, P.H. PDF receptor signaling in Drosophila contributes to both circadian and geotactic behaviors. Neuron 2005, 48, 213-219. [CrossRef] 
35. Lear, B.C.; Merrill, C.E.; Lin, J.M.; Schroeder, A.; Zhang, L.; Allada, R. A G protein-coupled receptor, groom-of-PDF, is required for PDF neuron action in circadian behavior. Neuron 2005, 48, 221-227. [CrossRef]

36. Kunst, M.; Tso, M.C.; Ghosh, D.D.; Herzog, E.D.; Nitabach, M.N. Rhythmic control of activity and sleep by class B1 GPCRs. Crit. Rev. Biochem. Mol. Biol. 2015, 50, 18-30. [CrossRef]

37. Johnson, E.C.; Shafer, O.T.; Trigg, J.S.; Park, J.; Schooley, D.A.; Dow, J.A.; Taghert, P.H. A novel diuretic hormone receptor in Drosophila: Evidence for conservation of CGRP signaling. J. Exp. Biol. 2005, 208, 1239-1246. [CrossRef]

38. Shafer, O.T.; Kim, D.J.; Dunbar-Yaffe, R.; Nikolaev, V.O.; Lohse, M.J.; Taghert, P.H. Widespread receptivity to neuropeptide PDF throughout the neuronal circadian clock network of Drosophila revealed by real-time cyclic AMP imaging. Neuron 2008, 58, 223-237. [CrossRef] [PubMed]

39. Kunst, M.; Hughes, M.E.; Raccuglia, D.; Felix, M.; Li, M.; Barnett, G.; Duah, J.; Nitabach, M.N. Calcitonin gene-related peptide neurons mediate sleep-specific circadian output in Drosophila. Curr. Biol. 2014, 24, 2652-2664. [CrossRef]

40. Goda, T.; Tang, X.; Umezaki, Y.; Chu, M.L.; Kunst, M.; Nitabach, M.N.N.; Hamada, F.N. Drosophila DH31 Neuropeptide and PDF Receptor Regulate Night-Onset Temperature Preference. J. Neurosci. 2016, 36, 11739-11754. [CrossRef]

41. Choi, C.; Fortin, J.P.; McCarthy, E.; Oksman, L.; Kopin, A.S.; Nitabach, M.N. Cellular dissection of circadian peptide signals with genetically encoded membrane-tethered ligands. Curr. Biol. 2009, 19, 1167-1175. [CrossRef] [PubMed]

42. Zhang, L.; Chung, B.Y.; Lear, B.C.; Kilman, V.L.; Liu, Y.; Mahesh, G.; Meissner, R.A.; Hardin, P.E.; Allada, R. $\mathrm{DN1}(\mathrm{p})$ circadian neurons coordinate acute light and PDF inputs to produce robust daily behavior in Drosophila. Curr. Biol. 2010, 20, 591-599. [CrossRef] [PubMed]

43. Tang, X.; Roessingh, S.; Hayley, S.E.; Chu, M.L.; Tanaka, N.K.; Wolfgang, W.; Song, S.; Stanewsky, R.; Hamada, F.N. The role of PDF neurons in setting the preferred temperature before dawn in Drosophila. Elife 2017, 6, e23206. [CrossRef]

44. Baines, R.A.; Uhler, J.P.; Thompson, A.; Sweeney, S.T.; Bate, M. Altered electrical properties in Drosophila neurons developing without synaptic transmission. J. Neurosci. 2001, 21, 1523-1531. [CrossRef]

45. Picot, M.; Klarsfeld, A.; Chelot, E.; Malpel, S.; Rouyer, F. A role for blind DN2 clock neurons in temperature entrainment of the Drosophila larval brain. J. Neurosci. 2009, 29, 8312-8320. [CrossRef]

46. Helfrich-Forster, C. The neuroarchitecture of the circadian clock in the brain of Drosophila melanogaster. Microsc. Res. Technol. 2003, 62, 94-102. [CrossRef]

47. Feinberg, E.H.; Vanhoven, M.K.; Bendesky, A.; Wang, G.; Fetter, R.D.; Shen, K.; Bargmann, C.I. GFP Reconstitution Across Synaptic Partners (GRASP) defines cell contacts and synapses in living nervous systems. Neuron 2008, 57, 353-363. [CrossRef]

48. Gordon, M.D.; Scott, K. Motor control in a Drosophila taste circuit. Neuron 2009, 61, 373-384. [CrossRef]

49. Kim, J.; Zhao, T.; Petralia, R.S.; Yu, Y.; Peng, H.C.; Myers, E.; Magee, J.C. mGRASP enables mapping mammalian synaptic connectivity with light microscopy. Nat. Methods 2012, 9, 96. [CrossRef] [PubMed]

50. Yao, Z.; Macara, A.M.; Lelito, K.R.; Minosyan, T.Y.; Shafer, O.T. Analysis of functional neuronal connectivity in the Drosophila brain. J. Neurophysiol. 2012, 108, 684-696. [CrossRef] [PubMed]

51. Tang, X.; Platt, M.D.; Lagnese, C.M.; Leslie, J.R.; Hamada, F.N. Temperature integration at the AC thermosensory neurons in Drosophila. J. Neurosci. 2013, 33, 894-901. [CrossRef]

52. Shih, H.W.; Chiang, A.S. Anatomical characterization of thermosensory AC neurons in the adult Drosophila brain. J. Neurogenet. 2011, 25, 1-6. [CrossRef]

53. Das, A.; Holmes, T.C.; Sheeba, V. dTRPA1 in Non-circadian Neurons Modulates Temperature-Dependent Rhythmic Activity in Drosophila melanogaster. J. Biol. Rhythms 2016. [CrossRef] [PubMed]

54. Yuan, Q.; Lin, F.; Zheng, X.; Sehgal, A. Serotonin modulates circadian entrainment in Drosophila. Neuron 2005, 47, 115-127. [CrossRef]

55. Morrison, S.F.; Nakamura, K. Central Mechanisms for Thermoregulation. Annu. Rev. Physiol. 2018. [CrossRef]

56. Tan, C.L.; Knight, Z.A. Regulation of Body Temperature by the Nervous System. Neuron 2018, 98, 31-48. [CrossRef] [PubMed] 
57. Cambras, T.; Weller, J.R.; Angles-Pujoras, M.; Lee, M.L.; Christopher, A.; Diez-Noguera, A.; Krueger, J.M.; de la Iglesia, H.O. Circadian desynchronization of core body temperature and sleep stages in the rat. Proc. Natl. Acad. Sci. USA 2007, 104, 7634-7639. [CrossRef] [PubMed]

58. Russell, F.A.; King, R.; Smillie, S.J.; Kodji, X.; Brain, S.D. Calcitonin gene-related peptide: Physiology and pathophysiology. Physiol. Rev. 2014, 94, 1099-1142. [CrossRef]

59. Kee, Z.; Kodji, X.; Brain, S.D. The Role of Calcitonin Gene Related Peptide (CGRP) in Neurogenic Vasodilation and Its Cardioprotective Effects. Front. Physiol. 2018, 9, 1249. [CrossRef] [PubMed]

60. Masi, L.; Brandi, M.L. Calcitonin and calcitonin receptors. Clin. Cases Miner. Bone Metab. 2007, 4, 117-122.

61. Nakamoto, H.; Soeda, Y.; Takami, S.; Minami, M.; Satoh, M. Localization of calcitonin receptor mRNA in the mouse brain: Coexistence with serotonin transporter mRNA. Brain Res. Mol. Brain Res. 2000, 76, 93-102. [CrossRef]

62. Becskei, C.; Riediger, T.; Zund, D.; Wookey, P.; Lutz, T.A. Immunohistochemical mapping of calcitonin receptors in the adult rat brain. Brain Res. 2004, 1030, 221-233. [CrossRef]

63. Doi, M.; Murai, I.; Kunisue, S.; Setsu, G.; Uchio, N.; Tanaka, R.; Kobayashi, S.; Shimatani, H.; Hayashi, H.; Chao, H.W.; et al. Gpr176 is a Gz-linked orphan G-protein-coupled receptor that sets the pace of circadian behaviour. Nat. Commun. 2016, 7, 10583. [CrossRef] [PubMed]

64. Abrahamson, E.E.; Moore, R.Y. Suprachiasmatic nucleus in the mouse: Retinal innervation, intrinsic organization and efferent projections. Brain Res. 2001, 916, 172-191. [CrossRef]

65. Shiromani, P.J.; Xu, M.; Winston, E.M.; Shiromani, S.N.; Gerashchenko, D.; Weaver, D.R. Sleep rhythmicity and homeostasis in mice with targeted disruption of mPeriod genes. Am. J. Physiol. Regul. Integr. Comp. Physiol. 2004, 287, R47-R57. [CrossRef] [PubMed]

66. Nagashima, K.; Matsue, K.; Konishi, M.; Iidaka, C.; Miyazaki, K.; Ishida, N.; Kanosue, K. The involvement of Cry1 and Cry2 genes in the regulation of the circadian body temperature rhythm in mice. Am. J. Physiol. Regul. Integr. Comp. Physiol. 2005, 288, R329-R335. [CrossRef] [PubMed]

67. Gerhart-Hines, Z.; Feng, D.; Emmett, M.J.; Everett, L.J.; Loro, E.; Briggs, E.R.; Bugge, A.; Hou, C.; Ferrara, C.; Seale, P.; et al. The nuclear receptor Rev-erbalpha controls circadian thermogenic plasticity. Nature 2013, 503, 410-413. [CrossRef]

68. Wolff, G.; Duncan, M.J.; Esser, K.A. Chronic phase advance alters circadian physiological rhythms and peripheral molecular clocks. J. Appl. Physiol. 2013, 115, 373-382. [CrossRef] [PubMed] 